\title{
Colonoscopy videos on YouTube: Are they a good source of patient education?
}

\section{(ㄷ)(1) $\odot$}

\section{Authors}

Dhruvil Radadiya ${ }^{1}$, Alexei Gonzalez-Estrada², Jorge Emilio Lira-Vera ${ }^{3}$, Katia Lizarrga-Torres ${ }^{4}$, Shayan Sinha Mahapatra ${ }^{5}$, Ricardo Murguia-Fuentes ${ }^{6}$, Sebastian Niezen? ${ }^{7}$ Kaushik Mohan ${ }^{8}$, Chakradhar Reddy $^{9}$, Kalpit Devani $^{9}$

\section{Institutions}

1 Department of Internal Medicine, East Tennessee State University, Johnson City, Tennessee, United States

2 Division of Allergy and Immunology, Department of Internal Medicine, Mayo Clinic Florida, Jacksonville, Florida, United States

3 Division of Gastroenterology, Hospital Español de México, Universidad Nacional Autónoma de México, Mexico City

4 Special Consultant, Private Practice, Panama City, Panama

5 Division of Endocrinology, Department of Internal Medicine, Cleveland Clinic, Florida, United States

6 Universidad Nacional Autónoma de Mexico, Mexico

7 Anahuac University School of Medicine, Mexico

8 University of Tennessee, Knoxville, Tennessee, United States

9 Division of Gastroenterology, Department of Internal Medicine, East Tennessee State University, Johnson City, Tennessee, United States

submitted 7.8.2019

accepted after revision $\quad 5.2 .2020$

Bibliography

DOI https://doi.org/10.1055/a-1122-8566 |

Endoscopy International Open 2020; 08: E598-E606

(c) Georg Thieme Verlag KG Stuttgart · New York elSSN 2196-9736

Corresponding author

Dhruvil Radadiya, MD, 325 N state of Franklin Road, Johnson City, TN, 37604

Fax: +1-423-439-6387

Dhruvil.Radadiya3@gmail.com

\# Supplementary material

Online content viewable at:

https://doi.org/10.1055/a-1122-8566

\section{ABSTRACT}

Background and study aims Colonoscopy is an effective tool to prevent colorectal cancer. Social media has emerged as a source of medical information for patients. YouTube (a video sharing website) is the most popular video informative source. Therefore, we aimed to assess the educational quality of colonoscopy videos available on YouTube.

Methods We performed a YouTube search using the keyword "colonoscopy" yielded 429 videos, of which 255 met the inclusion criteria. Colonoscopy Data Quality Score (CDQS) was created to rate the quality of the videos $(-10$ to +40 points) based on a colonoscopy education video available on the Ameican Society of Gastrointestinal Endoscopy (ASGE) website. Each video was scored by six blinded reviewers independently using C-DQS. The Global Quality Score (GQS) was used for score validation. The intraclass correlation coefficient (ICC) was used to assess the similarity of the scores among reviewers.

Results Professional societies had the highest number of videos (44.3\%). Videos from professional societies (6.94) and media (6.87) had significantly higher mean C-DQS compared to those from alternative medicine providers (1.19), companies (1.16), and patients (2.60) $(P<0.05)$. Mean C-DQS score of videos from healthcare providers (4.40) was not statistically different than other sources. There was a high degree of agreement among reviewers for the videos from all sources $($ ICC $=0.934 ; P<0.001)$.

Discussion YouTube videos are a poor source of information on colonoscopy. Professional societies and media are better sources of quality information for patient education on colonoscopy. The medical community may need to engage actively in enriching the quality of educational material available on YouTube. 


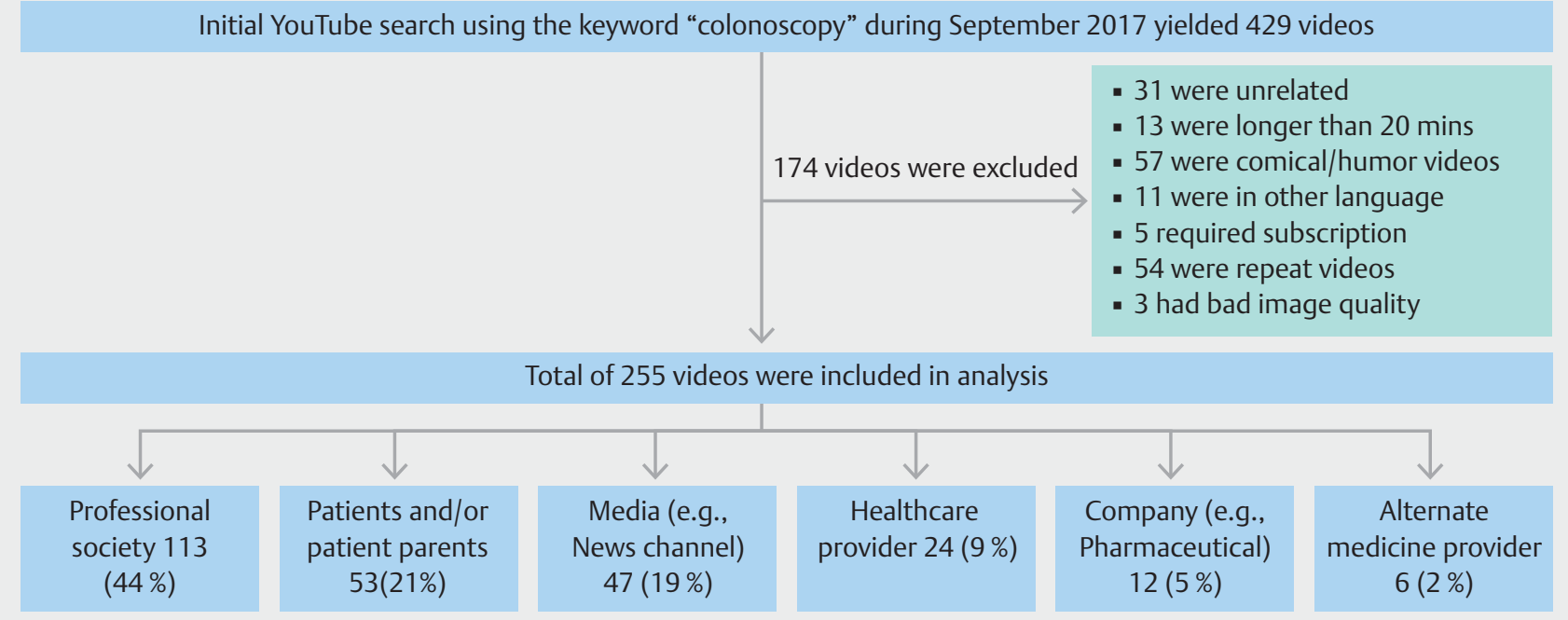

- Fig. 1 Study design.

\section{Introduction}

Colorectal cancer (CRC) is the third most common cancer and the second leading cause of cancer-related deaths among men and women in the United States [1]. Colonoscopy is one of the most effective screening methods to prevent CRC [2]. Being a therapeutic procedure, it has more perks than any other means of screenings. There are an estimated 14.2 million colonoscopies performed each year in the United States [3]. Still, the rate of screening colonoscopies among the general population remains suboptimal $[4,5]$. Despite its benefits, only $62.4 \%$ of men and women reported colorectal cancer screening test use consistent with the United States Preventive Services Task Force (USPSTF) recommendations [6].

In the last decade, social media has emerged as a key player in the dissemination of healthcare-related information among the general public to help them make informed medical decisions. YouTube, a video-sharing website, is one of the most popular video informative source on the internet [7-9]. Although it was created to provide entertainment videos initially, it can be a powerful learning tool for the general population. YouTube has more than 1.5 billion logged-in users per month and feeds over 1 billion hours of video each day to users [10, 11]. YouTube videos have been previously evaluated for healthcare-related information of various diseases and procedures and were reported to be of variable educational quality [1222]. This expanding trend of heavy reliance on the Internet may impact the patient-physician relationship and necessitates for a physician to have insight into the quality of such content. Colonoscopy being an invasive procedure with an intense bowel preparation regimen, it often provokes anxiety among patients, who then resort to the Internet in search of more information rather than contacting healthcare professionals. Therefore, we decided to assess the educational quality of colonoscopy videos available through different sources on YouTube. This study also highlights the important question of how the healthcare com- munity can embrace surge in social media reliance for procuring medical information to improve patient experience.

\section{Methods}

We performed a YouTube search during September 2017, using the keyword 'colonoscopy' to identify all available videos on colonoscopy since the inception of the YouTube website. We included videos in English, which lasted $\leq 20$ minutes and with good visual quality (defined as $\geq 240$ pixels of progressive scan). We excluded videos in languages other than English, unrelated to colonoscopy, repeat videos, or with poor visual quality, as shown in > Fig. 1.

Colonoscopy videos were analyzed for video characteristics and source. Video characteristics included sex and race depicted, number of views, number of likes and dislikes, number of comments, and duration. We categorized the video sources into healthcare provider, alternate medicine provider, patient and/or patient's parents, company (pharmaceutics mostly), media (news), and professional society. Alternate medicine was defined as treatments that are used instead of standard medical treatments (e. g., vidoes showing diets or colon hydrotherapy or herbal supplements to prevent colon cancer). We created a scoring system called "Colonoscopy Data Quality Score (C-DQS)" considering a colonoscopy education video [23] available on the American Society of Gastrointestinal Endoscopy (ASGE) website a gold standard and compared quality content of the other videos relative to it. Videos were rated with a starting score of 0 , with a total score that ranged from10 to 40 points ( $\downarrow$ Table $\mathbf{1}$ ). Points were subtracted for any misleading information (e.g. "colonoscopy increased risk of colon cancer," "colonoscopy is the useless procedure"). Points were awarded for accurate information covering all relevant topics as mentioned on ASGE website education video. We also scored each video by a previously published validated score system to 
- Table 1 Colonoscopy Data Quality Score (C-DQS).

Defines colonoscopy (1 point) e. g., colonoscopy is the procedure to look at large bowel, and it is a safe and effective procedure.

Mentions that colonoscopy is currently the best screening method for preventing colon cancer (1 point)

States prevalence of screening colonoscopy (1 point) e. g., the number of colonoscopies performed 14.2 million per year in the USA

Defines colonoscope (1 point) It is a flexible tube with a small camera and light at the end, which allows the examination of the colon.

Indications for colonoscopy as a diagnostic and therapeutic procedure (1 point each, maximum 5 points)

Colon cancer

Polyps

Hemorrhoids

IBD

Large intestinal disorders

Gives an estimate of colon cancer risk over the lifetime (1 point) e. g., Every individual has a $6 \%$ risk of developing colon cancer over a lifetime.

Mentions that Colonoscopy helps to prevent cancer by removing polyps before they turn into cancer (1 point)

Mentions that colonoscopy is the only test which can find and remove polyp at the same time among colon cancer screening tests (1 point)

\section{Expectation before the procedure:}

Mentions that the patient will be given written preparation instructions before the procedure (1 point)

Describes Bowel preparation regimen (1 point) e. g., Cleansing begins before the day of the exam, and there are different types of bowel prep

Mentions that clear liquid one day before the procedure should be followed (1 point)

Emphasizes the importance of good bowel prep better colon visualization and avoid cancellation (1 point) e. g., proper bowel prep means the quicker, safer, more comfortable, and more effective procedure.

Recommends no eating or drinking 6 hours before the procedure (1 point)

Describes that doctor will advise withholding certain medication before the procedure (1 point)

Encourages patients to ask a question to the medical team if needed ( 1 point)

\section{Expectation during the procedure:}

Mentions that Informed consent will be obtained after explaining risk, benefits, and alternatives (1 point)

Mentions that Procedure will be performed under sedation (1 point)

Mentions that for sedation Iv access will be obtained (1 point)

Describes procedure in general (1 point) e. g. Colonoscopy is done in a procedure room equipped with colonoscope and TV screen to see images of the colon.

Describes colonoscope and how does it work (1 point) e. g. Flexible tube with camera and light source at the end with a channel to pass instruments for biopsy. The scope has dials to maneuver in a different direction. The scope will be passed to the cecum.

Describes what endoscopist looking for during procedure (1 point) e. g., Polyps, source of bleeding, inflammation, etc.

Mentions that the duration of colonoscopy is usually less than an hour (1 point)

Mentions that colonoscopy is a well-tolerated procedure (1 point)

Describes complications of the procedure( 1 point each, maximum of 4 points)

Perforation

bleeding

missing polyp or lesion

adverse drug reaction

Defines polyp and/or types of polyp (1 point)

\section{Expectation after the procedure:}

Mentions that the patient will be closely watched for 30 mins after the procedure (1 point)

Mentions that patient will be made aware of preliminary results, new medications and follow up plan (1 point) 
$>$ Table 1 (Continuation)

Mentions that Mild bloating and gas expected after the procedure ( 1 point)

Mentions what is the biopsy and states that biopsy results can take several days and how to follow up (1 point)

Mentions that the patient should not go to work or drive or handle heavy machinery on the same day (1 point)

Mentions that the patient will need somebody to drive him/her back home (1 point)

Mentions that the patient can return to normal activity the next day following the procedure (1 point)

Advises the patient to call a doctor if the patient has abdominal pain, bleeding or fever (1 point)

Negative points:

Any false information such as included below but not limited to ( -1 point each):

Colonoscopy is useless procedure.

Colonoscopy increases risk of colon cancer.

Colonoscopy does not prevent colon cancer.

Colonoscopy does not have any supporting scientific evidence.

Colonoscopy should not be performed in asymptomatic patients.

Colonoscopy is a high-risk procedure with a high mortality rate and complication rate.

Colonoscopy is an expensive procedure.

Colonoscopy is performed without sedation and very uncomfortable.

Bowel preparation is not needed before colonoscopy.

Colonoscopy is a solely diagnostic procedure.

Score (maximum 40 points)

- Table2 Global Quality Score (GQS).

1 Poor quality, poor flow of the site, most information missing, not at all useful for patients

2 Generally poor quality and poor flow, some information listed but many important topics missing, of minimal use to patients

3 Moderate quality, suboptimal flow, some important information is adequately discussed, but others poorly explained, somewhat useful for patients

4 Good quality and generally good flow. Most of the relevant information is listed, but some topics not covered, useful for patients

$5 \quad$ Excellent quality and flow, beneficial for patients

rate the overall quality of health-care videos; global quality score (GQS) [24] outlined in > Table 2 . Six blinded medical reviewers agreed to evaluate all the videos. Each reviewer was educated to score video accurately with standard ASGE video and was provided contact information of the senior author (K. D.) if any questions arose throughout the review process. All the reviewers scored each video independently and were blinded to each other's scoring and comments.

This study was exempt from IRB approval as no patient-level data were used. REDcap (Research Electronic Data Capture) software was used to capture the scoring of videos and data tabulation [25]. Video characteristics were described using medians for all continues variables and counts as well as percentages for all categorical variables. All analyses were performed at a significant level of 0.05 . One Way-Analysis of variance was used to compare mean scores (GQS and C-DQS) by the video source. Post-hoc analysis using fisher's test of least significant difference was performed to evaluate the exact difference between mean scores, and sources were grouped accordingly (significance level set at $P<0.05$ ). The intraclass correlation coefficient (ICC) was used to assess the reliability of scoring between independent reviewers. Of note, an ICC score between 0.75 and 1.00 is considered excellent. Correlation between mean C-DQS and GQS was also done using a scatter plot and Pearson correlation coefficient. Correlation between mean CDQS and mean GQS, including scores of all videos was also assessed with the Pearson correlation coefficient. SAS 9.3 software (SAS Institute, Cary, NC) and R version 3.5.3 (R Foundation for Statistical Computing, Vienna, Austria) were used for all the analyses. 
- Table 3 Colonoscopy video characteristics.

\begin{tabular}{|l|r|}
\hline Variable & $\mathbf{N}=\mathbf{2 5 5}$ \\
\hline Sex depicted, no. (\%) & \\
\hline - Male & $114(44.7)$ \\
\hline - Female & $98(38.4)$ \\
\hline - Both & $40(17.3)$ \\
\hline Race Depicted, no. (\%) & $168(62.5)$ \\
\hline - White & $20(7.4)$ \\
\hline - African-American & $5(1.9)$ \\
\hline - Hispanic & $39(14.5)$ \\
\hline - Asian & $37(13.8)$ \\
\hline - None & 5503 \\
\hline Median no. views & 11 \\
\hline Median no. likes & 1 \\
\hline Median no. dislikes & 1 \\
\hline Median no. comments & 236 \\
\hline Median duration, seconds & \\
\hline
\end{tabular}

\section{Results}

Use of the keyword "colonoscopy" generated 429 videos, of which 174 were excluded based on our criteria. Two hundred fifty-five videos were included in the final analysis. The most common video source was professional societies (44\%). Baseline Video characteristics were summarized in $>$ Table 3. A higher proportion of video presenters were male $(42.5 \%)$. The most common depicted race of presenters was white $(62.5 \%)$. Videos had a median of 5503 views, 11 likes, and 1 dislikes, and had a median duration of 236 seconds. We also ranked the top 10 videos according to C-DQS score, most of them (75\%) were uploaded by professional societies. These videos, along with their links and video source, can be found in $>$ Table 4 . Overall mean scores for each video source was low, with mean C-DQS of all the videos 5.38 out of 40 points. Videos from professional societies had the highest mean C-DQS (6.94) and GQS (2.40) score, while videos from companies, mostly pharmaceuticals, had the lowest mean C-DQS (1.16) and GQS (1.25). Difference between scores of these two groups was statistically significant $(P<0.05)$. Both the scores are summarized by video source in - Fig. 2. Overall, there was a statistically significant difference in the mean C-DQS, and GQS score among videos by the video source $(P<0.001)$ as per one way-ANOVA test. Post hoc analysis

- Table 4 Top 10 videos by C-DQS.

\begin{tabular}{|c|c|c|c|c|c|c|}
\hline Rank & Video Link & Video Source & Video Source Type & $\begin{array}{l}\text { Number } \\
\text { of likes }\end{array}$ & $\begin{array}{l}\text { Duration } \\
\text { (min:sec) }\end{array}$ & $\begin{array}{l}\text { Mean } \\
\text { C-DQS }\end{array}$ \\
\hline 1 & $\begin{array}{l}\text { https://www.youtube.com/ } \\
\text { watch?v=eA1PIMa1ULg }\end{array}$ & $\begin{array}{l}\text { The American Society for } \\
\text { Gastrointestinal Endoscopy }\end{array}$ & Professional society & 253 & 10:08 & 40 \\
\hline 2 & $\begin{array}{l}\text { https://www.youtube.com/ } \\
\text { watch?v=Eqs2HLQdLEY }\end{array}$ & $\begin{array}{l}\text { UW Medicine, Division of } \\
\text { Gastroenterology }\end{array}$ & Professional society & 218 & 18:19 & 26.1 \\
\hline 3 & $\begin{array}{l}\text { https://www.youtube.com/ } \\
\text { watch?v=5b0D3zVpjlU }\end{array}$ & $\begin{array}{l}\text { Lourdes Health and Medical } \\
\text { Center }\end{array}$ & Professional Society & 4 & 10:05 & 24.6 \\
\hline 4 & $\begin{array}{l}\text { https://www.youtube.com/ } \\
\text { watch?v=gp9yHYwbO7U }\end{array}$ & CDHFtube by Dr. Alan Barkun & Health care provider & 9 & $12: 09$ & 23.3 \\
\hline 5 & $\begin{array}{l}\text { https://www.youtube.com/ } \\
\text { watch?v=b5j-NCvOpjs }\end{array}$ & AGA Institute & Professional society & 576 & 10:03 & 22.8 \\
\hline 6 & $\begin{array}{l}\text { https://www.youtube.com/ } \\
\text { watch?v=pQ-eq93Znls }\end{array}$ & $\begin{array}{l}\text { NorthShore University } \\
\text { HealthSystem }\end{array}$ & Professional society & 69 & $6: 34$ & 20.6 \\
\hline 6 & $\begin{array}{l}\text { https://www.youtube.com/ } \\
\text { watch?v=Z3sZ4XysGsA }\end{array}$ & $\begin{array}{l}\text { Bumrungrad International } \\
\text { Hospital }\end{array}$ & Professional society & 6 & $6: 15$ & 20.6 \\
\hline 7 & $\begin{array}{l}\text { https://www.youtube.com/ } \\
\text { watch?v=mh90RPA-C10 }\end{array}$ & $\begin{array}{l}\text { www.YouAndColonoscopy. } \\
\text { com }\end{array}$ & Media & 1580 & $5: 14$ & 19.1 \\
\hline 8 & $\begin{array}{l}\text { https://www.youtube.com/ } \\
\text { watch?v=lorHwnC6JkY }\end{array}$ & $\begin{array}{l}\text { South West Regional Cancer } \\
\text { Program }\end{array}$ & Professional society & 0 & 9:30 & 18.1 \\
\hline 9 & $\begin{array}{l}\text { https://www.youtube.com/ } \\
\text { watch?v=UOiiNfiU3K0 }\end{array}$ & MD Anderson Cancer Center & Professional society & 193 & $8: 07$ & 18 \\
\hline 9 & $\begin{array}{l}\text { https://www.youtube.com/ } \\
\text { watch?v=w6Dn7KRjpLs }\end{array}$ & Vancouver Coastal Health & Professional society & 0 & $8: 35$ & 18 \\
\hline 10 & $\begin{array}{l}\text { https://www.youtube.com/ } \\
\text { watch?v=YhBloQpEiZA }\end{array}$ & Easy Health & Media & 12 & $10: 54$ & 16.8 \\
\hline
\end{tabular}




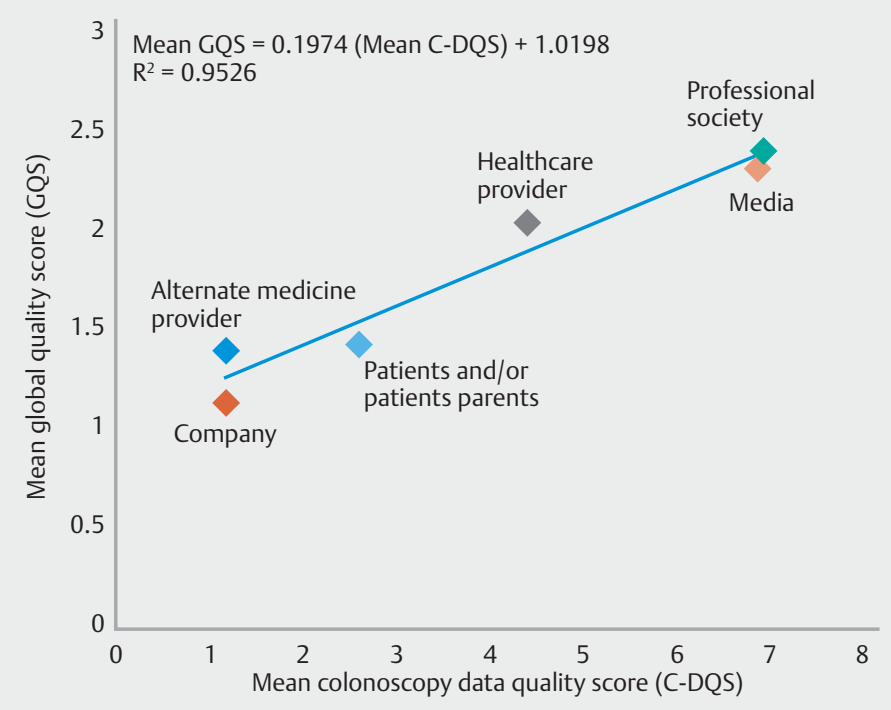

\begin{tabular}{l|c|c}
\hline Video source & C-DQS & GQS \\
\hline Professional society & 6.94 & 2.40 \\
\hline Media (e.g., news channels) & 6.87 & 2.31 \\
\hline Healthcare provider & 4.40 & 2.03 \\
\hline Patient and/or patient parents & 2.60 & 1.43 \\
\hline Alternative medicine provider & 1.19 & 1.39 \\
\hline Company (e.g., Pharmaceuticals) & 1.16 & 1.25 \\
\hline
\end{tabular}

Number presented in table are mean C-DQS (colonoscopy data quality score) and mean GQS (global quality score).

- Fig. 2 Correlation between mean C-DQS and GQS.

- Table 5 Difference between mean C-DQS and mean GQS among video sources.

\begin{tabular}{|c|c|c|c|c|}
\hline Video source & Mean C-DQS ${ }^{1}$ & Grouping $^{2}$ & Mean GQS ${ }^{1}$ & Grouping $^{2}$ \\
\hline Professional society & 6.94 & a & 2.40 & a \\
\hline Media & 6.87 & a & 2.31 & a \\
\hline Healthcare provider & 4.40 & $a b$ & 2.03 & $b$ \\
\hline Patient and/or patient parents & 2.60 & $b$ & 1.43 & b \\
\hline Alternative medicine provider & 1.19 & b & 1.39 & $b$ \\
\hline Company & 1.16 & b & 1.25 & $b$ \\
\hline \multicolumn{5}{|c|}{$\begin{array}{l}\text { C-DQS, colonoscopy data quality score; GQS, global quality score } \\
1 \text { One-way ANOVA showed that mean GQS, and C-DQS among sources were not same }(P<0.0001) \\
2 \text { Grouping of source based on fisher's test of least significance (significance set at } P<0.05) \text {. Each same lower case letter shows that there is no difference in mean } \\
\text { C-DQS/GQS between two sources with same letter. Two lowercase letters assigned to a source suggest the no difference in mean score with either of group re- } \\
\text { presented by individual letter. }\end{array}$} \\
\hline
\end{tabular}

to assess the difference between individual video sources using fisher's test of least significant difference (LSD) showed that there was no difference in mean C-DQS scores between profession society and media but individual mean of both sources individually was different from mean C-DQS of patient and/or patient parents, company and alternative medicine provider $(P<0.05)$. We grouped video sources based on the difference in mean C-DQS and GQS as evident from > Table 5, which made comparison easy to follow. The differences of mean CDQS and GQS between individuals video source, standard deviation, and interquartile range can be seen in Supplementary Table 1, Supplementary Table 2, Supplementary Table 3 and Supplementary Table 4.

Correlation between mean C-DQS and GQS was performed using a scatter plot, which yielded correlation coefficient (R) 0.975 , which predicts a strong correlation. Coefficient of determination $\left(R^{2}\right)$ was 0.952 , which means that $95 \%$ of the total var- iance in mean GQS can be explained by the linear relationship between mean C-DQS and GQS. Additionally, we performed a Pearson correlation test, which yielded a correlation coefficient of $0.981(P<0.0005)$ confirming the strong correlation. Mean GQS and CDQS by all videos were also compared to evaluate the association between this two scoring system, as shown in Supplementary Fig.1, which showed a strong correlation (Pearson correlation coefficient $=0.932, P<0.0001$ ). There was a high degree of correlation among the reviewers (Intraclass correlation coefficient [ICC]: 0.934; $P<0.001)$. Each video source individually was consistently rated by individual reviewers as evident from ICC presented in $>$ Table 6 except for videos from companies which had ICC $<0.75$, representing moderate consistency. 
- Table6 Mean colonoscopy data quality score by video source.

\begin{tabular}{|l|l|l|l|l|}
\hline Video source & Mean & ICC & Confidence Interval & P value \\
\hline All sources & 5.38 & 0.934 & $0.908-0.951$ & $<0.001$ \\
\hline $\begin{array}{l}\text { Professional society (e. g., hospital, organization, } \\
\text { healthcare society) }\end{array}$ & 6.94 & 0.933 & $0.894-0.956$ & 255 \\
\hline Media (e. g., news channel) & 6.87 & 0.922 & $0.863-0.956$ & $<0.001$ \\
\hline Health care provider (e. g., physician) & 4.40 & 0.946 & $0.904-0.974$ & $<0.001$ \\
\hline Patient and/or patient's parents & 2.60 & 0.859 & $0.788-0.912$ & $<0.001$ \\
\hline Company (e. g., pharmaceutical company) & 1.16 & 0.546 & $0.003-0.848$ & $<0.001$ \\
\hline Alternative medicine provider & 1.19 & 0.840 & $0.487-0.975$ & 0.026 \\
\hline \begin{tabular}{l} 
ICC, intraclass correlation co-efficient; N, number of videos \\
\hline
\end{tabular} & & & 0.001 \\
\hline
\end{tabular}

\section{Discussion}

This study analyzed 255 colonoscopy videos available on YouTube, the most popular video hosting site accessible through the Internet to the general population [7,8]. Overall analysis showed that YouTube remains a poor information source regarding colonoscopy. Professional societies and Media had highest C-DQS and GQS compared to other sources seem to be spreading quality information on this topic. Multiple studies with similar pretext have been done for various medical conditions, and in agreement, to those, our study also shows that YouTube is a poor and unreliable source of medical information $[1,7,12-22,26]$.

The Videos from professional societies, the most common video source in our study, received both the highest mean GQS score and C-DQS score. However, the overall mean was still significantly lower than the upper limit of C-DQS, suggesting variable quality among the individual videos. We noticed frequent use of medical jargon in some of the videos, which can fail to attract the viewers and may disinterest them after a while. The same can apply to videos uploaded by health care providers, which were ranked third on C-DQS and were the fourth most common video source. Also, there was wide range of variability in the scores of those videos. The videos being uploaded from individual or institution related to medicine does not guarantee quality information. Two studies, one on videos on femoroacetabular impingement and one on asthma, both of which included a significant number of videos from healthcare providers, concluded that information available was inadequate in terms of overall quality $[13,26]$. Therefore, medical professionals should be more cautious to ensure the videos being uploaded are in plain language and should be available without subscription so a wide population can gain information from them. Also, there is a growing need for the medical community to participate in actively creating such education material and making it accessible to the general population.

Media, mostly composed of news channel, were the secondhighest ranked video source on C-DQS and had a comparable mean score to a professional society, suggesting video quality in par with professional society. There may be multiple factors behind this. Katie Couric's live colonoscopy on national television bumped up the colonoscopy rate in the general population, which was sustained for 9 months [27]. This shows that brief and accurate information provided by public figure can have a tremendous amount of effect in public opinion. Also, videos uploaded by media often avoid medical terminology, which is a significant barrier for laypersons. However, media can move people in a different direction with controversial statements to increase their viewers, which always remains a concern. But for now, it seems that news media is spreading quality content in the community in terms of colonoscopy education.

The second most common video source - patients and/or their parents - fails to touch on essential aspects of the procedure. These videos were created with the pure intention of attracting the audience rather than spreading information and debunking common misconception of procedure, which is evident from the low scoring on both scales, with overall quality comparable to the lowest-ranked video sources (Company and Alternative medicine provider). Patients and/or family members can serve as a guide to other people regarding what would be the procedure like and what to expect in terms of preparation, particularly bowel prep. We noticed that some of the high-quality videos by professional societies and/or healthcare providers included patients who shared their experiences briefly, which increased the overall quality of the videos.

Alternative Medicine videos scored somewhat better than sources like companies and patient/patient parents but had overall poor quality compared to professional society and media. Although they represented a small proportion of videos in our analysis, sometimes they attract more viewers, due to controversial and misleading information. Another reason behind this can be a growing trend of preference for alternative medicine. The information they provide does not have enough scientific support and is not considered the standard of care. We came across the following statements while watching these videos: 1) "Colonoscopy may actually increase risk of colon cancer"; 2) "Colonoscopy is useless"; 3) "Colonoscopy is not needed if you don't have any symptoms"; 4) "Women don't need colonoscopy"; 5) "Colonoscopy is nothing but a hoax so doctors 
can earn money and there is no scientific literature backing up colonoscopy for screening of colorectal cancer"; and 6) "Risk associated with colonoscopy outweighs the benefits." Healthcare providers should be vigilant and should educate patients when they came across such videos.

Videos from companies, mostly pharmaceutical companies, ranked the lowest on both scales, suggesting overall poor education quality. They were focused more on advertisements for different preparation agents rather than the procedure as a whole. Also, some videos were meant to be for medical professionals specifically, to attract them to a particular type of colonoscope to improve visualization of polyps and help with maneuverability. Pharmaceutical companies as such do not have a role in terms of colonoscopy education, but they can help patients with proper bowel preparation by making videos dedicated to that part only.

Social media is a two-edged sword. Depending upon the use and user, it can be a bliss or a curse in the healthcare field. While videos uploaded by professional societies and healthcare providers undergo some kind of review process before being uploaded on social media, videos from other sources do not. This can be a source of medical misinformation, which can lead to patient harm. This should compel us to form a team of medical experts who can moderate such medical content on the Internet. While we understand that the Internet represents a vast source of information and not everything can be moderated, we believe there is a need for a team of trained medical professionals, who can periodically assess major sources like YouTube, Twitter, and Facebook and report and/or remove healthcare content with misinformation. In addition, we as healthcare providers can direct patients to sources of high educational quality focused on education rather than misinformation.

There were some limitations in our study. Being a retrospective study, it has inherent bias related to such study design. Our study findings cannot be generalized to YouTube videos in a language other than English, longer than 20 minutes or that have poor image quality. YouTube is a dynamic website with videos uploaded each day, but this is the first large study evaluating more than 250 videos on colonoscopy. Interobserver variability can be a concern with the study design, but as evident from ICC, we can predict there was consistent reliability among reviewers in terms of scoring. Finally, people with medical backgrounds evaluated the videos, so it is not possible to assess how a general non-medical population would score the videos or how health literacy might impact scoring.

\section{Conclusion}

Colonoscopy videos on YouTube remain a poor source of evidence-based quality information. Our study shows that the general population should rely on videos uploaded by professional societies and media if they chose to use YouTube to get more information on colonoscopy. The medical community needs to be proactive to increase awareness and use social media to counter misconceptions that the general population may have about CRC screening.

\section{Competing interests}

The authors declare that they have no conflict of interest.

\section{References}

[1] Wong M, Desai B, Bautista M et al. YouTube is a poor source of patient information for knee arthroplasty and knee osteoarthritis. Arthroplast Today 2019; 5: 78-82

[2] Rex DK, Boland CR, Dominitz JA et al. Colorectal cancer screening: recommendations for physicians and patients from the U.S. MultiSociety Task Force on Colorectal Cancer. Am J Gastroenterol 2017; 112: $1016-1030$

[3] Seeff LC, Richards TB, Shapiro JA et al. How many endoscopies are performed for colorectal cancer screening? Results from CDC's survey of endoscopic capacity Gastroenterology 2004; 127: 1670-1677

[4] Davis MM, Renfro S, Pham R et al. Geographic and population-level disparities in colorectal cancer testing: A multilevel analysis of Medicaid and commercial claims data. Prev Med 2017; 101: 44-52

[5] Vijan S, Inadomi J, Hayward RA et al. Projections of demand and capacity for colonoscopy related to increasing rates of colorectal cancer screening in the United States. Aliment Pharmacol Ther 2004; 20 : 507-515

[6] White A, Thompson TD, White MC et al. Cancer screening test use United States, 2015. MMWR Morb Mortal Wkly Rep 2017; 66: 201206

[7] Vance K, Howe W, Dellavalle RP. Social Internet sites as a source of public health information. Dermatol Clin 2009; 27: 133-136, vi

[8] Alexa global rankings. Available from (Accessed 04 June 2019) https://www.alexa.com/siteinfo/youtube.com

[9] Perrin A. Share of U.S. adults using social media, including Facebook, is mostly unchanged since 2018. 2019: Available from (Accessed 04 June 2019) https://www.pewresearch.org/fact-tank/2019/04/10/ share-of-u-s-adults-using-social-media-including-facebook-is-mostly-unchanged-since-2018|

[10] Davies D. Meet the 7 most popular search engines in the world. 2018: Available from: Accessed 04 June 2019 https://www.searchenginejournal.com/seo-101/meet-search-engines/

[11] Blog YO. You know what's cool? A billion hours 2017: Available from (Accessed 04 June 2019) https://youtube.googleblog.com/2017/02/ you-know-whats-cool-billion-hours.html

[12] Yaylaci S, Serinken M, Eken C et al. Are YouTube videos accurate and reliable on basic life support and cardiopulmonary resuscitation? Emerg Med Australas 2014; 26: 474-477

[13] MacLeod MG, Hoppe DJ, Simunovic $N$ et al. YouTube as an information source for femoroacetabular impingement: a systematic review of video content. Arthroscopy 2015; 31: 136-142

[14] Lee JS, Seo HS, Hong TH. YouTube as a source of patient information on gallstone disease. World J Gastroenterol 2014; 20: 4066-4070

[15] Sorensen JA, Pusz MD, Brietzke SE. YouTube as an information source for pediatric adenotonsillectomy and ear tube surgery. Int J Pediatr Otorhinolaryngol 2014; 78: 65-70

[16] Syed-Abdul S, Fernandez-Luque L, Jian WS et al. Misleading healthrelated information promoted through video-based social media: anorexia on YouTube. J Med Internet Res 2013; 15: e30

[17] Mukewar S, Mani P, Wu X et al. YouTube, and inflammatory bowel disease. J Crohns Colitis 2013; 7: 392-402

[18] Briones R, Nan X, Madden $\mathrm{K}$ et al. When vaccines go viral: an analysis of HPV vaccine coverage on YouTube. Health Commun 2012; 27: $478-485$ 
[19] Stamelou M, Edwards M], Espay A] et al. Movement disorders on YouTube-caveat spectator. N Engl J Med 2011; 365: 1160-1161

[20] Richardson CG, Vettese L, Sussman S et al. An investigation of smoking cessation video content on YouTube. Subst Use Misuse 2011; 46 893-897

[21] Steinberg PL, Wason S, Stern JM et al. YouTube as source of prostate cancer information. Urology 2010; 75: 619-622

[22] Brooks FM, Lawrence $H$, Jones A et al. YouTube as a source of patient information for lumbar discectomy. Ann R Coll Surg Engl 2014; 96 : 144-146

[23] American Society of Gastrointestinal Endoscopy. Colonoscopy: What Patients Can Expect. 2009: access from: https://www.youtube.com/ watch?v=eA1PIMa1ULg
[24] Bernard A, Langille M, Hughes S et al. A systematic review of patient inflammatory bowel disease information resources on the World Wide Web. Am J Gastroenterol 2007; 102: 2070-2077

[25] Harris PA, Taylor R, Thielke R et al. Research electronic data capture (REDCap) - a metadata-driven methodology and workflow process for providing translational research informatics support. J Biomed Inform 2009; 42: 377-381

[26] Gonzalez-Estrada A, Cuervo-Pardo L, Ghosh B et al. Popular on YouTube: a critical appraisal of the educational quality of information regarding asthma. Allergy Asthma Proc 2015; 36: e121-126

[27] Cram P, Fendrick AM, Inadomi I et al. The impact of a celebrity promotional campaign on the use of colon cancer screening: the Katie Couric effect. Arch Intern Med 2003; 163: 1601-1605 